\title{
Blockade of angiotensin II type-1 receptor increases salt sensitivity in Sprague-Dawley rats
}

\author{
Satoshi Endo ${ }^{1}$, Takefumi Mori ${ }^{1,2}$, Yoshimi Yoneki ${ }^{1}$, Takashi Nakamichi ${ }^{1}$, Takuma Hosoya ${ }^{1}$, Susumu Ogawa ${ }^{1}$, \\ Goro Tokudome ${ }^{3}$, Tatsuo Hosoya ${ }^{3}$, Toshio Miyata ${ }^{4}$ and Sadayoshi Ito ${ }^{1}$
}

This study determined the role of angiotensin II type-1 (AT1) receptor in the salt sensitivity of blood pressure. The mean arterial blood pressure (MAP) of Sprague-Dawley rats was monitored by radio telemetry and, after baseline measurements, rats were treated either with (1) vehicle, (2) AT1 receptor blocker (ARB) olmesartan (OLM, $100 \mathrm{nmol} \mathrm{kg}^{-1} \mathrm{~h}^{-1}$, subcutaneously), (3) OLM with hydrochlorothiazide (HCTZ, $40 \mathrm{mg} \mathrm{kg}^{-1}$ day $^{-1}$, orally), (4) angiotensin II (AngII, $100 \mathrm{ng} \mathrm{kg}^{-1} \mathrm{~min}^{-1}$, subcutaneously) or with (5) AngII with OLM. Rats were fed a $0.5 \%$ salt diet during the baseline and first 7 days of treatment period, and the diet was then switched to one containing $8 \%$ salt for another 7 days. Urinary samples were collected in a metabolic cage at the end of each period. MAP of the vehicle group did not change throughout the study. In Angll-infused rats, BP increased only when rats were fed an $8 \%$ salt diet. OLM and OLM with Angll significantly reduced MAP when rats were on a $0.5 \%$ salt diet, but not on an $8 \%$ salt diet, indicating an enhanced salt sensitivity by OLM. Co-treatment with HCTZ reduced the salt sensitivity of OLM. The urinary level of the oxidative stress marker was increased by an $8 \%$ salt diet and was not altered by either OLM alone or in combination with HCTZ. However, OLM attenuated the salt-induced renal NAD(P)H (nicotinamide adenine dinucleotide phosphate) oxidase activity. These results indicate that AT1 receptor blockade increases salt sensitivity, which is reversed by diuretics. We conclude that OLM and HCTZ could be a useful combination for reduction of blood pressure even under high salt intake without changes in urinary oxidative stress levels.

Hypertension Research (2009) 32, 513-519; doi:10.1038/hr.2009.40; published online 1 May 2009

Keywords: angiotensin II; angiotensin receptor blockers; diuretics; salt sensitivity

\section{INTRODUCTION}

Hypertension is an independent risk factor of cardiovascular diseases (CVD). ${ }^{1}$ The renin-angiotensin system (RAS) blockers are now parts of major antihypertensive treatments for protection against hypertensive vascular and renal injuries. ${ }^{2,3}$ It is to be noted that the antihypertensive effects of RAS blockers are abrogated by high salt loading in hypertensive individuals ${ }^{4}$ and in experimental animal models., ${ }^{5,6}$ Therefore, salt restriction or combination therapy with diuretics is recommended to those who do not respond well with RAS inhibitors alone. ${ }^{7-9}$

RAS regulates urinary excretion of water and sodium, thereby determining blood pressure. Hall et al. ${ }^{10}$ confirmed this chronic pressure natriuresis in dogs: both angiotensin II (AngII) and angiotensin-converting enzyme inhibitor, SQ-14225, altered the relationship between salt intake and blood pressure, thereby affecting the salt sensitivity of blood pressure. Recently, several types of angiotensin receptors have been identified and their signaling pathways have been elucidated. ${ }^{11}$ Nevertheless, the role of an angiotensin receptor in the salt sensitivity of blood pressure remains to be fully investigated.
This study was thus undertaken to test the hypothesis that the blockade of AngII type 1 (AT1) receptor alters the salt sensitivity of blood pressure in the presence or absence of a diuretic. For detailed analysis of blood pressure, including diurnal variations, mean arterial blood pressure (MAP) was continuously monitored by 24 -h radio telemetry in Sprague-Dawley rats, given AT1 receptor blockers (ARB) and/or diuretics, while changing their salt intake. In another model, AngII was infused subcutaneously to suppress endogenous AngII and to keep high circulating levels of AngII. Finally, we investigated a link of salt sensitivity to oxidative stress in rats that were given ARB and/or diuretics, as both salt intake and AngII have impacts on oxidative stress, a factor involved in the initiation and progression of hypertensive cardiovascular and renal injuries. ${ }^{12,13}$

\section{METHODS}

Animals

Male Sprague-Dawley rats (SLC, Shizuoka, Japan), 8-10-weeks old, were fed a $0.5 \% \mathrm{NaCl}$ diet (Labo MR Standard, Nosan Corporation Biodepartment,

\footnotetext{
${ }^{1}$ Division of Nephrology, Endocrinology and Vascular Medicine, Tohoku University Graduate School of Medicine, Tohoku University, Sendai, Japan; ${ }^{2}$ Health Administration Center, Tohoku University, Sendai, Japan; ${ }^{3}$ Division of Nephrology and Hypertension, Jikei University School of Medicine, Jikei University, Minato-ku, Tokyo and ${ }^{4}$ Center for Translational and Advanced Research, Tohoku University Graduate School of Medicine, Tohoku University, Sendai, Japan

Correspondence: Dr T Mori, Division of Nephrology, Endocrinology and Vascular Medicine, Tohoku University Graduate School of Medicine, Tohoku University, 1-1 Seiryocho, Aoba-ku, Sendai 980-8574, Japan.

E-mail: tmori2i@mail.tains.tohoku.ac.jp

Received 6 November 2008; revised 13 February 2009; accepted 13 March 2009; published online 1 May 2009
} 
Kanagawa, Japan) before the study. Tap water was provided ad libitum throughout the study. The rats were housed in controlled conditions (temperature: $20^{\circ} \mathrm{C}$; lighting $8-20 \mathrm{~h}$ ). All procedures were in accordance with the National Research Council's guidelines and were approved by the institutional animal care committee.

Experiment 1: Effect of salt intake on blood pressure in rats treated with angiotensin II, AT1 receptor blocker or with the AT1 receptor blocker in combination with diuretics

Rats were anesthetized with $30 \mathrm{mg} \mathrm{kg}^{-1}$ of ketamine and with $2 \mathrm{mg} \mathrm{kg}^{-1}$ of xylasine. A radio-telemetry transmitter (PA-C40 Data Science International, Primetec, Tokyo, Japan) was implanted in the left femoral artery and the body of the transmitter was placed subcutaneously at the side of abdomen. Buprenorphine at $0.09 \mathrm{mg} \mathrm{kg}^{-1}$ was administered subcutaneously for analgesia during recovery from the surgery. The rats were allowed to recover for 5-7 days before the study. The rats were divided into five groups: (1) vehicle (Veh), (2) olmesartan (OLM), (3) olmesartan with hydrochlorothiazide (OLM+HCTZ), (4) AngII and (5) AngII with olmesartan (AngII+OLM). Veh, OLM and AngII were delivered using an osmotic mini-pump (Alzet Model 2002, Muromachi, Tokyo, Japan), which was implanted subcutaneously and infused at a rate of $0.5 \mu \mathrm{h}^{-1}$. HCTZ (Sigma-Aldrich, Tokyo, Japan) was given by gavage ( $40 \mathrm{mg} \mathrm{kg}^{-1} \mathrm{day}^{-1}$ in $0.5 \%$ carboxymethylcellulose). Olmesartan (provided by Sankyo Pharmaceutical Company, Tokyo, Japan) was dissolved in $2.5 \%$ sodium carbonate, and infused at $100 \mathrm{nmol} \mathrm{kg}^{-1} \mathrm{~h}^{-1}$. AngII (Sigma-Aldrich), dissolved in saline, was infused at $100 \mathrm{ng} \mathrm{kg}^{-1} \mathrm{~min}^{-1}$. The Veh group received sodium carbonate using the osmotic mini-pump. An osmotic mini-pump was implanted subcutaneously at the back of the neck during a brief procedure using ether anesthesia. The rats were fed a $0.5 \%$ salt diet (normal salt) for 7 days and then switched to an $8 \%$ salt (high salt) diet for another 7 days. 24-h urine was collected in a metabolic cage on the final day of the $0.5 \%$ salt baseline pressure measurements and the $8 \%$ salt period. Urinary sodium concentration was measured using the UniCel DxC 800 Synchron Clinical System (Beckman Instruments, Tokyo, Japan).

\section{Blood pressure analysis}

Blood pressure data were measured at $500 \mathrm{~Hz}$ for $10 \mathrm{~s}$ every $3 \mathrm{~min}$. MAP was averaged over the last two days of each diet period for determination of the chronic steady-state pressure-natriuresis relationship. The diurnal rhythm of pressure was also analyzed. The averages of pressures from 6:00-8:00 am, which reflected the highest pressures of the day, were used to represent nighttime (dark phase) blood pressure. The average of pressures from 6:00-8:00 pm, which reflected the lowest pressures of the day, were used as daytime (light phase) blood pressure.

\section{Estimation of salt sensitivity}

Salt sensitivity was estimated from the slope of relationship between $\mathrm{Na}$ excretion and blood pressure using the following equation: Na-blood pressure slope $=($ urinary sodium excretion (on an $8 \%$ salt diet $)$-urinary sodium excretion (on a $0.5 \%$ salt diet))/(MAP (on an $8 \%$ salt diet)-MAP (on a $0.5 \%$ salt diet)). If the slope of the relationship between $\mathrm{Na}$ excretion and blood pressure is lower, the result was indicated as higher salt sensitivity.

\section{Experiment 2: Effect of angiotensin II receptor blocker on urinary} markers of oxidative stress

Male SD rats, 8 -weeks old, fed a $0.5 \%$ salt diet for more than 7 days before the study were divided into 10 study groups: (1) and (6) vehicle, (2) and (7) OLM, (3) and (8) OLM with HCTZ (OLM+HCTZ), (4) and (9) AngII and (5) and (10) AngII with OLM (AngII+OLM). (1)-(5) groups were fed a $0.5 \%$ salt diet, whereas (6)-(10) groups were fed an $8 \%$ salt diet. Veh, OLM and AngII were delivered using the osmotic mini-pump implanted subcutaneously for 7 days as described in Experiment 1. The rats were housed in a metabolic cage for 24-h urine collection in a bottle maintained below $4{ }^{\circ} \mathrm{C}$, at the baseline and at the 7 days after the diet was changed to an $8 \%$ salt diet. Urinary $\mathrm{H}_{2} \mathrm{O}_{2}$ concentrations were determined with the reaction of resorufin in the presence of horseradish peroxidase with an Amplex Red reagent (10-acetyl-3,7dihydroxyphenoxazine, Molecular Probes, Tokyo, Japan), according to the manufacturer's instructions. $\mathrm{H}_{2} \mathrm{O}_{2}$ levels in all samples were expressed as a catalase-inhibitable fraction. As the absolute baseline urinary levels of oxidative stress were variable, the results were expressed and compared as fold increase from baseline level.

\section{Experiment 3: Effect of AT1 receptor blockade on enhanced $\mathrm{NAD}(\mathrm{P}) \mathrm{H}$ oxidase activity by high salt intake}

To determine whether OLM would attenuate enhanced $\mathrm{NAD}(\mathrm{P}) \mathrm{H}$ (nicotinamide adenine dinucleotide phosphate) oxidase activity in response to a high salt intake, 8 -weeks-old male SD rats were fed a $0.5 \%$ salt diet for more than 7 days before the study and divided into four groups: (1) vehicle with rats fed a $0.5 \%$ salt diet (vehicle-NS), (2) vehicle with rats fed an $8 \%$ salt diet (vehicleHS), (3) OLM with rats fed a $0.5 \%$ salt diet (OLM-NS) and (4) OLM with rats fed an $8 \%$ salt diet (OLM-HS). Vehicle and OLM were delivered with osmotic mini-pumps implanted subcutaneously for 7 days as described in Experiment 1. After the rats were fed either a $0.5 \%$ or an $8 \%$ salt diet for 7 days, each was anesthetized with pentobarbital $\left(50 \mathrm{mg} \mathrm{kg}^{-1}\right.$ body weight, IP) and the left kidney was removed for measuring the $\mathrm{NAD}(\mathrm{P}) \mathrm{H}$ oxidase activity. The renal cortex was separated and immediately frozen under liquid nitrogen and kept at $-80^{\circ} \mathrm{C}$ before the assay.

The renal cortical tissue $\mathrm{NAD}(\mathrm{P}) \mathrm{H}$ oxidase activity was measured according to the methods of Guo et al. ${ }^{14}$ The renal cortical tissue $(50-100 \mathrm{mg})$ was transferred into test tubes containing $1 \mathrm{ml}$ of phosphate-buffered saline. The samples were homogenized on ice and centrifuged at 3000 r.p.m. for $10 \mathrm{~min}$. The supernatant was used for further analysis. The protein concentrations of the supernatant were measured using the Bradford method (Bio-Rad Protein Assay, Tokyo, Japan) to normalize the tissue content. Lucigenin (final concentration $10 \mu \mathrm{moll}^{-1}$; Sigma-Aldrich)-enhanced chemiluminescence was used to determine superoxide anion generation. The increase in chemiluminescence in response to $100 \mu \mathrm{moll}^{-1}$ of $\mathrm{NAD}(\mathrm{P}) \mathrm{H}$ was measured. The diphenyleneiodonium ( $25 \mu \mathrm{moll}^{-1}$; Sigma-Aldrich)-inhibitable chemiluminescence signal was recorded using a tube luminometer (ATTO, Tokyo, Japan) to reflect the tissue $\mathrm{NAD}(\mathrm{P}) \mathrm{H}$ oxidase activity.

\section{Statistical analysis}

Values are expressed as mean \pm standard error. Blood pressure was evaluated using a two-way ANOVA (analysis of variance) with repeated measurements. A post hoc Bonferroni's $t$-test was carried out to determine the significance between groups. Urinary $\mathrm{H}_{2} \mathrm{O}_{2}$ excretion and the slope of the relationship between $\mathrm{Na}$ excretion and blood pressure were evaluated using an unpaired $t$-test. Significance was accepted at a level of $P<0.05$.

\section{RESULTS}

Effects of salt intake on blood pressure in rats treated with angiotensin II AT1 receptor blocker, or with the AT1 receptor blocker in combination with diuretics

As shown in Figure 1a, $24 \mathrm{~h}$ averages of MAP in the vehicle group did not significantly change from a baseline level of $92.8 \pm 2.8$ and $94.7 \pm 2.6 \mathrm{~mm} \mathrm{Hg}$ on the $0.5 \%$ salt diet compared with $103.0 \pm 3.4 \mathrm{~mm} \mathrm{Hg}$ on the $8 \%$ salt diet. OLM significantly reduced MAP from $97.1 \pm 3.3$ to $79.4 \pm 2.8 \mathrm{~mm} \mathrm{Hg}$ in rats fed a $0.5 \%$ salt diet. Reduction of MAP with OLM was diminished when rats were fed an $8 \%$ diet, and returned to a MAP level of $102 \pm 3.0 \mathrm{~mm} \mathrm{Hg}$. OLM administered with HCTZ lowered MAP from $92.1 \pm 1.7$ to $76.0 \pm 1.8 \mathrm{~mm} \mathrm{Hg}$ in rats fed a $0.5 \%$ salt diet, and MAP remained lower than that in the vehicle or OLM group even on the $8 \%$ salt diet $(89.9 \pm 3.6 \mathrm{~mm} \mathrm{Hg}$ ) (Figure 1a). AngII infused subcutaneously at $100 \mathrm{ng} \mathrm{kg}^{-1} \mathrm{~min}^{-1}$ did not significantly increase MAP (106.2 \pm 3.4 $\mathrm{mm} \mathrm{Hg}$ ) in rats fed the $0.5 \%$ salt diet (Figure $1 \mathrm{~b}$ ). However, AngII significantly increased MAP when rats were fed an $8 \%$ salt diet $(126.2 \pm 6.4 \mathrm{~mm} \mathrm{Hg})$. OLM administered with AngII reduced MAP from $98.6 \pm 2.1$ to $81.0 \pm 2.1 \mathrm{~mm} \mathrm{Hg}$ on the $0.5 \%$ salt diet, but returned to the vehicle control pressure levels when the rats were fed an $8 \%$ salt diet $(107.6 \pm 2.8 \mathrm{~mm} \mathrm{Hg})$. 

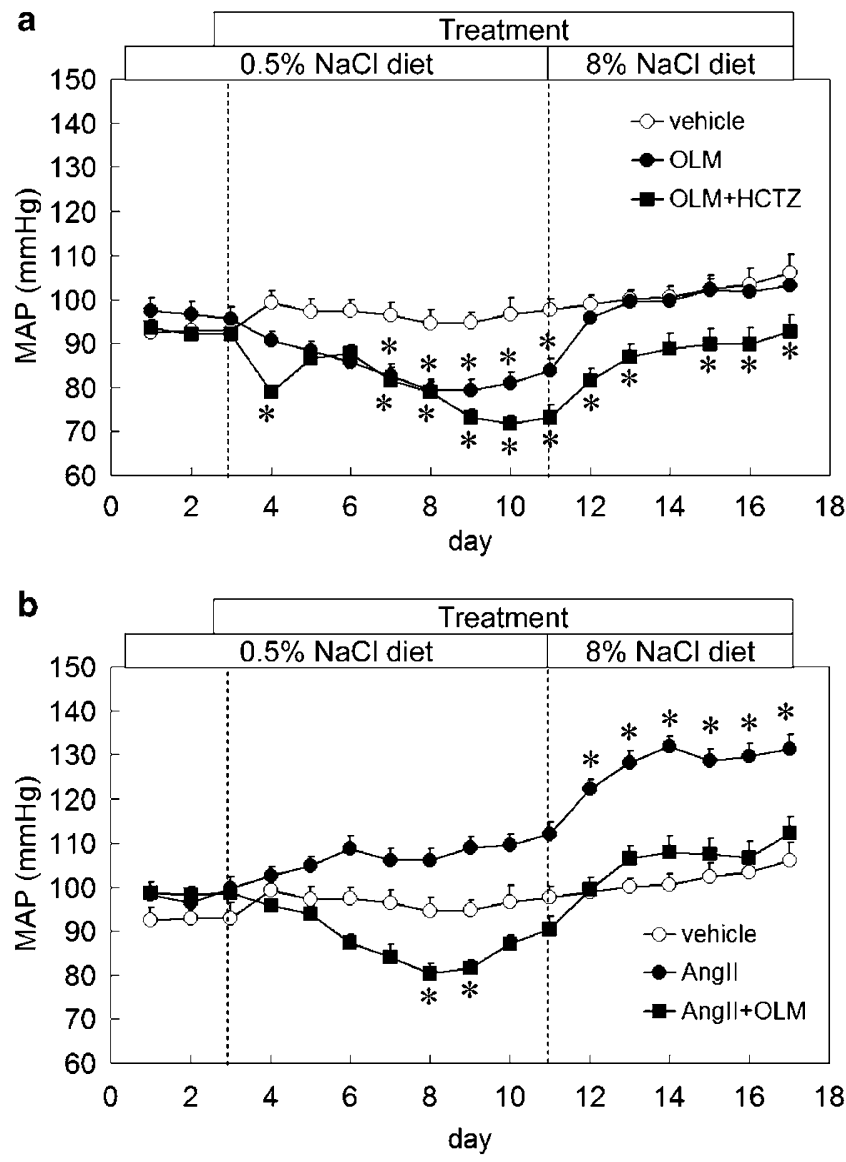

Figure 1 Daily changes in mean arterial pressure monitored by the radio telemetry system. An average of $24 \mathrm{~h}$ was plotted. (a) Comparison between vehicle $(n=6)$, olmesartan (OLM, $n=6)$ and OLM with the hydrochlorothiazide (OLM+HCTZ, $n=10)$ group. ${ }^{*} P<0.05$ vs. vehicle. (b) Comparison between vehicle $(n=6)$, angiotensin II (Ang II, $n=7$ ) and Ang II with the OLM (AngII+OLM, $n=6$ ) group. ${ }^{*} P<0.05$ vs. vehicle.

Salt sensitivity in rats treated with angiotensin II, AT1 receptor blocker or with the AT1 receptor blocker in combination with diuretics

To estimate salt sensitivity, the relationships between salt intake and the steady-state levels of MAP achieved (Na-MAP relationship) were determined and plotted in Figure 2. Salt sensitivity as indicated by the slope of the Na-MAP relationship is presented in Table 1. Changes of blood pressure induced by a high salt intake were miniscule in the Veh group, and the slope of the Na-MAP relationship was steep. The Na-MAP relationship of the AngII-infused group tended to be blunted, indicating that salt sensitivity is increased by AngII infusion. Although the level of blood pressure was shifted to a lower level, the slope of the Na-MAP relationship was significantly blunted in rats treated with OLM and in the group treated with OLM and AngII compared with that of the Veh group, indicating that AT1 receptor blockade increases salt sensitivity. By contrast, the Na-MAP relationship of the OLM group in which HCTZ was co-administered was steeper when compared to rats treated with OLM alone, indicating that the addition of HCTZ restored the salt sensitivity to normal.

\section{Circadian variation of MAP}

On a normal $0.5 \%$ salt diet, treatment with OLM significantly decreased MAP during both daytime and nighttime as compared

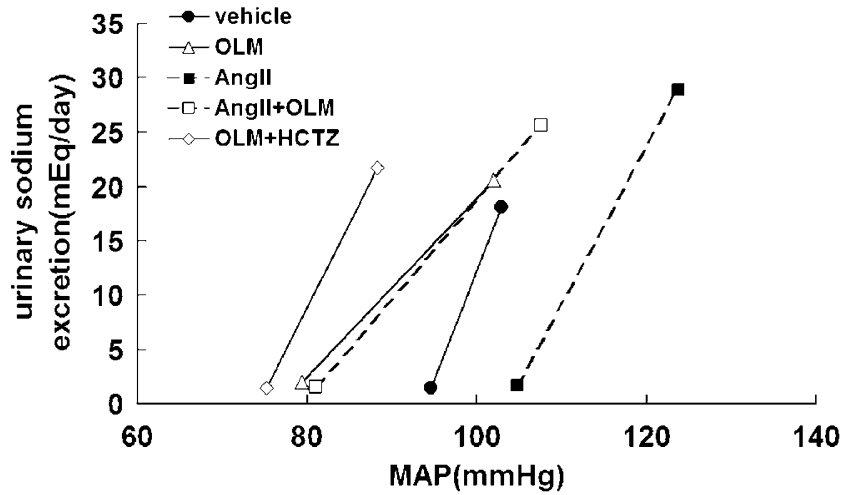

Figure 2 The Na-MAP relationship curve in vehicle $(n=6)$, olmesartan (OLM, $n=6)$, OLM with hydrochlorothiazide (OLM+HCTZ, $n=10$ ), angiotensin II (AngII, $n=7$ ) and Angll with OLM (AngII+OLM, $n=6$ ). The slope of the Na-MAP relationship curve was decreased in OLM, Ang II and Ang $\mathrm{II}+\mathrm{OLM}$.

with that in vehicle-treated rats (Figure 3a). By contrast, OLM treatment did not reduce MAP in the daytime or nighttime in rats fed a high $8 \%$ salt diet. Co-administration of OLM and HCTZ in rats did not significantly reduce MAP compared with that on administration of OLM alone, either in the daytime or nighttime, when rats were fed a $0.5 \%$ salt diet (Figure $3 \mathrm{~b}$ ). However, MAP was significantly reduced during the daytime in rats fed the high $8 \%$ salt diet. AngII administered together with OLM significantly reduced MAP during both daytime and nighttime in rats on a normal $0.5 \%$ salt diet as compared with that with AngII alone, but reduced MAP only in the daytime in rats fed a high $8 \%$ salt diet (Figure 3c). AngII administration alone diminished the reduction of daytime MAP, whether rats were fed the $0.5 \%$ or the high $8 \%$ salt diet, when compared with vehicle-treated rats (Figures $3 \mathrm{a}$ and $\mathrm{c}$ ).

\section{Effects of the AT1 receptor blocker on urinary markers of oxidative} stress

To determine whether overall body oxidative stress would be reduced by treatment with ARBs and diuretics, urinary $\mathrm{H}_{2} \mathrm{O}_{2}$ excretion was analyzed. As shown in Figure 4a, urinary $\mathrm{H}_{2} \mathrm{O}_{2}$ excretion, expressed as fold increase from the average baseline level, tended to increase in the AngII group than in vehicle-treated rats receiving a $0.5 \%$ salt diet $(1.68 \pm 0.48$ vs. $0.87 \pm 0.10(P=0.059))$. OLM rats, in which HCTZ was co-administered, exhibited significantly increased urinary $\mathrm{H}_{2} \mathrm{O}_{2}$ excretion than those of the vehicle group on a $0.5 \%$ salt diet $(2.38 \pm 0.56 v s$. $0.87 \pm 0.10$ times from baseline, $P<0.05)$. The AngII-with-OLMtreated group showed only a tendency for lower values when compared with those of the AngII-treated group (1.03 $\pm 0.28 \mathrm{vs}$. $1.68 \pm 0.48(P=0.32)$ on a $0.5 \%$ salt diet. Urinary $\mathrm{H}_{2} \mathrm{O}_{2}$ excretion significantly increased in all groups in rats fed the $8 \%$ salt diet compared with those on a $0.5 \%$ salt diet. OLM treatment was not able to inhibit the urinary marker of oxidative stress when rats were fed a high-salt diet and this was not different when OLM was used in combination with HCTZ.

Effect of AT1 blockade on enhanced $\mathrm{NAD}(\mathrm{P}) \mathrm{H}$ oxidase activity by high salt intake

As $\mathrm{NAD}(\mathrm{P}) \mathrm{H}$ oxidase has been shown to be stimulated with a highsalt diet, ${ }^{13}$ we determined whether renal $\mathrm{NAD}(\mathrm{P}) \mathrm{H}$ oxidase activity was influenced by a high-salt diet in the presence of AT1 receptor blockade. The results showed that renal cortical $\mathrm{NAD}(\mathrm{P}) \mathrm{H}$ oxidase 
Table 1 Salt sensitivity estimated from the slope of sodium excretion and MAP relationship

\begin{tabular}{|c|c|c|c|c|c|}
\hline & \multicolumn{2}{|c|}{ Sodium excretion (mEq per day) } & \multicolumn{2}{|c|}{$M A P(m m ~ H g)$} & \multirow[b]{2}{*}{ Slope } \\
\hline & NS & HS & NS & $H S$ & \\
\hline OLM & $1.9 \pm 0.2$ & $20.6 \pm 2.2$ & $79.4 \pm 0.8^{*}$ & $102.0 \pm 0.2$ & $0.83 \pm 0.10^{*}$ \\
\hline $\mathrm{OLM}+\mathrm{HCTZ}$ & $1.5 \pm 0.1$ & $23.4 \pm 2.5$ & $75.2 \pm 2.0^{*}$ & $86.7 \pm 3.6^{*}$ & $1.69 \pm 0.32$ \\
\hline All & $1.7 \pm 0.3$ & $28.8 \pm 2.4^{*}$ & $104.8 \pm 3.5$ & $123.7 \pm 6.7^{*}$ & $1.52 \pm 0.30$ \\
\hline
\end{tabular}

Abbreviations: HS, high salt; NS, normal salt.

Values are means \pm s.e.m. $(n=6)$.

$* P<0.05$ vs. vehicle.

a

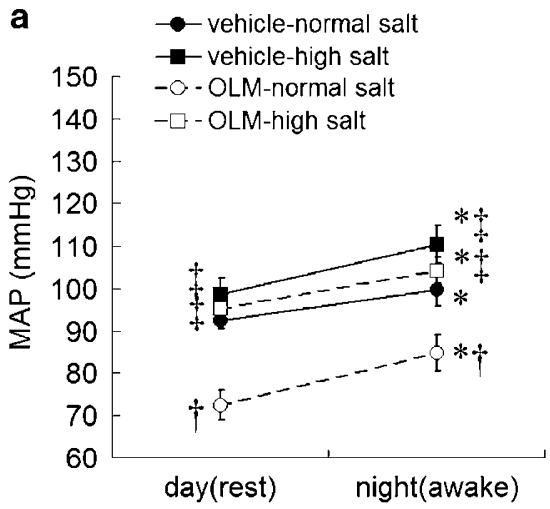

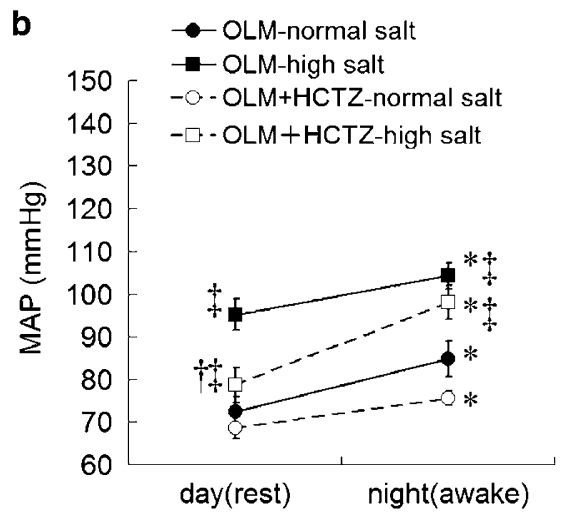

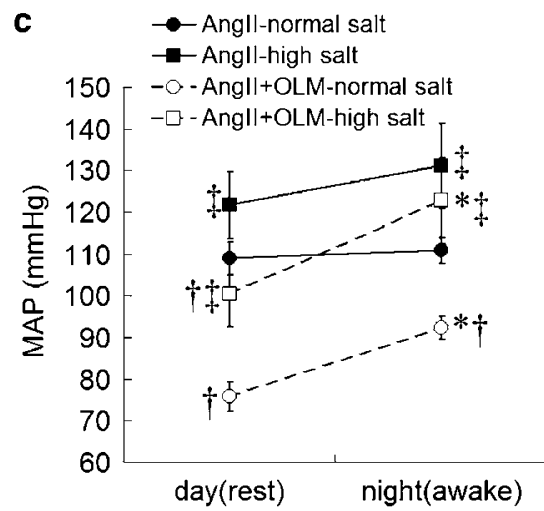

Figure 3 The diurnal rhythm of the mean arterial blood pressure (MAP). The lowest daytime and peak nighttime pressures are plotted. (a) Diurnal variations of the vehicle $(n=6)$ and olmesartan (OLM, $n=6)$ groups. ${ }^{*} P<0.05$ vs. daytime of MAP. ${ }^{\dagger} P<0.05$ vs. vehicle. ${ }^{\ddagger} P<0.05$ vs. normal salt. (b) Diurnal variations of $\mathrm{OLM}(n=6)$ and $\mathrm{OLM}$ with the hydrochlorothiazide (OLM+HCTZ, $n=10)$ group. ${ }^{\star} P<0.05$ vs. daytime of MAP. ${ }^{\dagger} P<0.05$ vs. OLM. ${ }^{\ddagger} P<0.05$ vs. normal salt. (c) Diurnal variations of angiotensin II (Ang II, $n=7$ ) and Ang II with the OLM ( $n=6)$ group. ${ }^{*} P<0.05$ vs. daytime of MAP. ${ }^{\dagger} P<0.05$ vs. Angll. $\ddagger P<0.05$ vs. normal salt.

activity was increased when rats were fed a high $8 \%$ salt diet, and that this was reduced by OLM treatment (Veh-NS, $139.0 \pm 11.3$; Veh-HS, $203.6 \pm 22.2$; OLM-HS $164.4 \pm 13.3 \times 10^{3}$ c.p.m. per $\mathrm{mg}$ protein) (Figure $4 \mathrm{~b}$ ). The enhanced renal $\mathrm{NAD}(\mathrm{P}) \mathrm{H}$ oxidase activity stimulated by a high $8 \%$ salt diet could therefore be reduced by AT1 receptor blockade.

\section{DISCUSSION}

The major finding of this study was that blockade of angiotensin II type-1 (AT1) receptors increased salt sensitivity in Sprague-Dawley rats. A combination of angiotensin II receptor blocker (ARB) and diuretics significantly reduced salt sensitivity and enhanced the antihypertensive effect of ARB in rats fed a high-salt diet. To the best of our knowledge, this study successfully determined salt sensitivity by $24 \mathrm{~h}$ blood pressure monitoring for the first time using the radiotelemetry transmitter. A high-salt intake increased both urinary oxidative stress and renal $\mathrm{NAD}(\mathrm{P}) \mathrm{H}$ oxidase activity. Although ARB, either combined with or without diuretics, did not alter high-saltinduced urinary oxidative stress, ARB attenuated the salt-induced $\mathrm{NAD}(\mathrm{P}) \mathrm{H}$ oxidase activity in the renal cortex. As shown in the clinical study by Vogt et al. ${ }^{9}$ it has been widely accepted that a low-salt diet reduces blood pressure during ARB treatment. However, there are not many clinical studies that have determined the association of salt sensitivity with ARB. As there are lots of parameters that could influence salt sensitivity in human clinical studies, such as body mass index, renal function, food intake and exercise, and may not easily administer angiotensin II receptor blockers and/or angiotensin II in normal subjects, this study was conducted in rats in a wellcontrolled condition.

\section{Altered salt sensitivity by AT1 receptor blockade}

The relationship between $\mathrm{Na}$ intake and blood pressure (Na-MAP relationship) is determined by the relationship between chronic $\mathrm{Na}$ excretion, which chronically equals the $\mathrm{Na}$ intake, and the steady-state level of MAP at any given level of salt intake. ${ }^{15}$ Salt sensitivity can be estimated by the reduction in the slope of the relationship between arterial pressure (MAP plotted on the abscissa) and sodium excretion (UNaV plotted on the ordinate), as reported by Kimura et al. ${ }^{16}$ The role of AngII on the chronic steady-state relationship between MAP and sodium excretion and sodium intake was first determined by Hall et al. ${ }^{10}$ in dogs given a continuous infusion of AngII. In these studies, the slope of the pressure-natriuresis relationship was reduced by chronic elevations of AngII, indicating an increase of salt sensitivity as arterial pressure significantly increased above normal at each new level of salt intake. It is interesting that in animals treated with an angiotensin-converting enzyme inhibitor, arterial pressure was reduced significantly below normal when dogs received a low-salt intake, but MAP increased with a slope similar to that observed in the AngII-infused dogs as daily salt intake was increased. These studies showed that angiotensin-converting enzyme increased salt sensitivity, whereby arterial pressure was reduced with sodium depletion and significantly increased in response to a high salt intake. ${ }^{10}$ The results of 

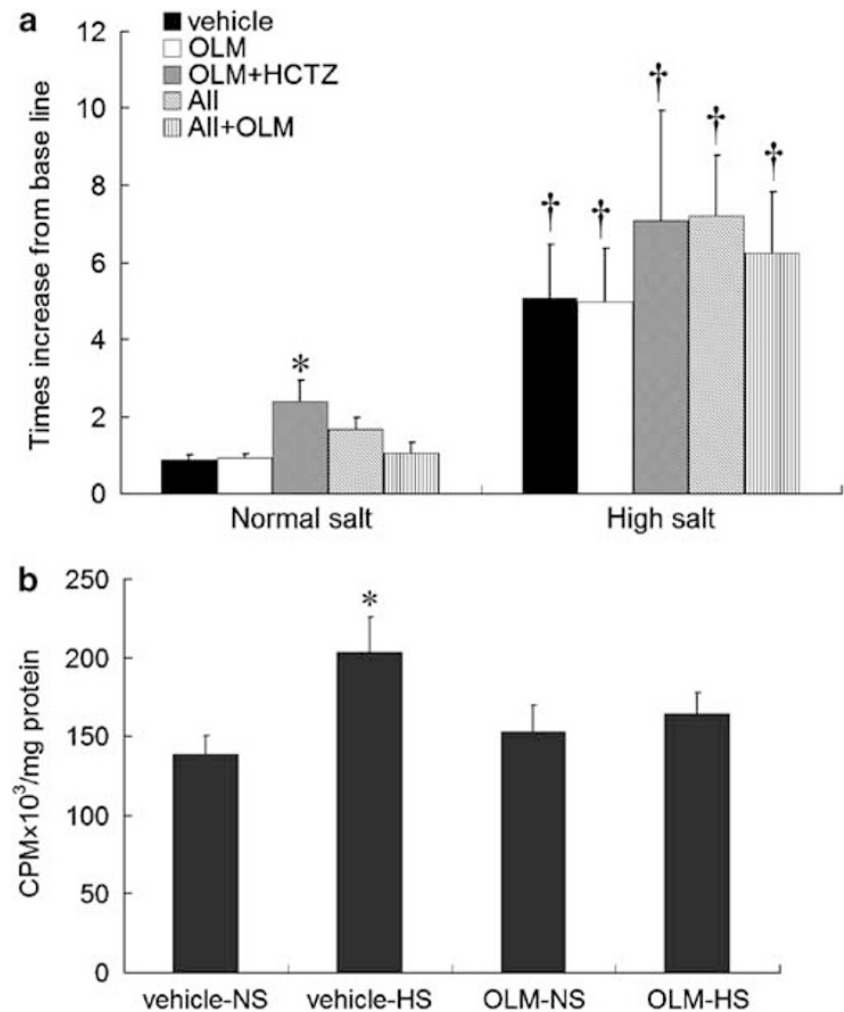

Figure 4 Oxidative stress induced by angiotensin II type-1 (AT1) receptor stimulation and a high-salt diet. (a) Fold increase of urinary $\mathrm{H}_{2} \mathrm{O}_{2}$ level from baseline in vehicle $(n=6)$, olmesartan (OLM, $n=6)$, OLM with hydrochlorothiazide (OLM+HCTZ, $\mathrm{n}=6)$, angiotensin $\|(A n g \|, n=6)$ and Angll with the OLM (AngII+OLM, $n=6$ ) group. ${ }^{*} P<0.05$ vs. Angll group. ${ }^{\dagger} P<0.05$ vs. $0.5 \%$ salt diet. (b) $\mathrm{NAD}(\mathrm{P}) \mathrm{H}$ oxidase activity of renal cortical tissue isolated from the vehicle (vehicle-NS, $n=6$ ) and olmesartan (OLM-NS, $n=6$ ) groups under a $0.5 \%$ salt diet, and from vehicle (vehicle-HS, $n=6$ ) and olmesartan (OLM-HS, $n=6$ ) groups under an $8 \%$ salt diet. ${ }^{*} P<0.05$ vs. the vehicle-NS group.

this study confirm the studies of Hall et al. in that AngII infusion shifted the Na-MAP relationship curve toward a higher pressure and the slope of the curve was reduced. Administration of the ARB also shifted the curve toward lower levels of pressure as seen by the reduction in the slope of the pressure-natriuresis relationship. Although patients with higher salt sensitivity of blood pressure normally have high blood pressure, the increased salt sensitivity by ARB in this study did not necessarily increase blood pressure. The results in this study show that ARB increases salt sensitivity by reducing blood pressure with lesser salt intake. Thus, it has been widely acknowledged in clinical practice that ARB reduces blood pressure with lower salt intake compared with higher salt intake.

The mechanism involved in the association between ARB and salt sensitivity is still not fully understood. We have shown that ARB reduced the slope of the Na-MAP relationship and increased salt sensitivity. As described in an earlier study, the Na-MAP relationship will become blunted and similar to acute pressure natriuresis when the angiotensin II level was fixed by angiotensin II infusion or angiotensin-converting enzyme inhibitor treatment. ${ }^{10}$ Not only the fixed angiotensin II level but also the continuous AT1 receptor blockade could blunt the Na-MAP relationship curve and increase salt sensitivity. However, this may not be the case with a pathological state, such as salt-sensitive hypertension, wherein ARB may reduce salt sensitivity. It has been shown that an increase in renal NO, which is also seen with RAS blockade, reduces blood pressure in high-salt-fed saltsensitive Dahl rats, ${ }^{17}$ indicating that $\mathrm{ARB}$ may have a possible mechanism that could reduce salt sensitivity in the salt-sensitive form of hypertension.

The action of AngII through various angiotensin receptors, such as angiotensin type 1 (AT1) and 2 (AT2), has been shown to be different. ${ }^{11}$ Only the AT1 receptors exerted any measurable influence on salt sensitivity in our studies as shown by observations that the Na-MAP relationship in rats with AT1 blockade was not different whether AngII was infused or not. These results are consistent with earlier studies showing that salt sensitivity was increased in $\mathrm{AT}_{1 \mathrm{~A}}$ receptor-deficient mice. ${ }^{18}$ Furthermore, Gross et al. also found that salt sensitivity was not enhanced in AT2 receptor-deficient mice. ${ }^{19}$ Taken together with the results of this study, we conclude that it is the AT1 receptor that plays a key role in the regulation of the Na-MAP relationship and salt sensitivity.

An important finding is that treatment with the combination of HCTZ and OLM resulted in a reduction of salt sensitivity, as seen by the recovery of the blunted slope of the $\mathrm{Na}-\mathrm{MAP}$ relationship in OLM-treated rats. The results of this study indicate that the antihypertensive effects of ARB therapy would be enhanced not only by the restriction of $\mathrm{Na}$ intake but also by a diuretic that reduced the reabsorption of filtered $\mathrm{Na}$, as shown in the clinical study. ${ }^{9}$

\section{Role of AT1 blockade to diurnal blood pressure rhythm}

Salt-sensitive hypertensive patients have been found to exhibit an altered diurinal pattern of arterial pressure, whereby the fall of nocturnal blood pressure is small ('non-dippers'), compared with salt-insensitive individuals. ${ }^{20}$ RAS plays an important role in nocturnal blood pressure in rats ${ }^{21}$ and humans. ${ }^{22,23}$ It has been shown that ARB administration in 'non-dipper' patients return the diurnal pattern to normal ${ }^{23,24}$ The results in this study have shown that AngII increased blood pressure during the resting phase and also increased blood pressure salt sensitivity. ARB administration reduced blood pressure during the resting phase of the diurnal cycle. Taken together, we propose that AT1 receptor stimulation is one of the determinants of the non-dipper pattern in the salt-sensitive form of hypertension. However, as shown in the clinical study by Fukuda et al. ${ }^{25}$ olmesartan administered in the morning once a day could restore nocturnal reduction of blood pressure. As this was associated with an increase in diurnal natriuresis, they have proposed that natriuresis, rather than RAS inhibition, is responsible for the reduction of nocturnal blood pressure by olmesartan. This is why we have determined renal and urinary oxidative stress, as renal oxidative stress increases $\mathrm{Na}$ reabsorption in renal tubules as we have previously reviewed. ${ }^{26}$ Although olmesartan did not inhibit urinary oxidative stress, significant inhibition was observed in renal oxidative stress. Therefore, this study supports the hypothesis that olmesartan increases natriuresis and restores nighttime BP by reducing renal oxidative stress. However, the question remains to be resolved whether this is dependent on the AT1 receptor.

OLM administered with HCTZ did not significantly decrease MAP compared with OLM treatment alone in either the resting or active phase of the diurinal cycle in rats fed a normal-salt diet. On the other hand, OLM co-administered with HCTZ significantly decreased MAP only in the resting phase on a high-salt diet. This observation is in accordance with clinical observations showing that diuretic therapy also changes the blood pressure diurinal rhythm from that of 'non-dipper' to 'dipper' in patients with essential hypertension. ${ }^{27}$ 
The mechanism whereby diuretic therapy affects nocturnal blood pressure has been reviewed by Uzu et al., ${ }^{27}$ who showed that the nocturnal fall in blood pressure is dependent on the amount of $\mathrm{Na}$ excreted in the daytime. These relationships, and the underlying mechanisms determining these diurnal blood pressure rhythms in normal and hypertensive rats, remain to be determined.

\section{Oxidative stress under AT1 receptor blockade on high-salt diet}

A number of studies have shown the role of oxidative stress and renal $\mathrm{NAD}(\mathrm{P}) \mathrm{H}$ oxidase in the progression of salt-sensitive hypertension and renal injury. ${ }^{28-30} \mathrm{~A}$ high salt intake enhanced urinary excretion of $\mathrm{H}_{2} \mathrm{O}_{2}$ in this study and it was not further increased by AngII administration. Consistent with these findings, Pech et al. ${ }^{31}$ observed that urinary 8-isoprostaglandin F2 alpha (8-ISO) was increased by AngII in rats fed a normal-salt diet and was further increased on a high-salt diet, but was not further increased by AngII. The results in this study and in the study by Kitiyakara et al. ${ }^{13}$ indicate that renal $\mathrm{NAD}(\mathrm{P}) \mathrm{H}$ oxidase activity is enhanced by a high salt intake. The discrepancy between urinary and renal oxidative stress markers may be explained by differences in the major site of oxidative stress. That is, we have only determined the renal cortical $\mathrm{NAD}(\mathrm{P}) \mathrm{H}$ oxidase activity whereas urinary oxidative stress may depend more importantly on medullary oxidative stress and/or systemic oxidative stress. It is also possible that other pathways related to free radical production may have contributed to the observed changes in $\mathrm{H}_{2} \mathrm{O}_{2}$ excretion. For example, Pech et al. ${ }^{31}$ have reported that the $\mathrm{NAD}(\mathrm{P}) \mathrm{H}$ oxidase inhibitor, apocynin, failed to reduce urinary 8-ISO levels that had been induced by a high salt intake.

Although this study did not determine a single effect of HCTZ to oxidative stress, it has been shown by Reungjui et al. ${ }^{32}$ that HCTZ treatment in rats increases renal cortical oxidative stress. We have previously shown in a clinical study that the thiazide diuretic, trichlormethiazide, failed to reduce urinary albumin excretion and oxidative stress in patients with diabetic nephropathy. ${ }^{33}$ Thus, it does not seem to follow that diuretics have a beneficial role in oxidative stress. In contrast, OLM when co-administered with HCTZ in rats fed a high-salt diet did not further increase urinary $\mathrm{H}_{2} \mathrm{O}_{2}$ excretion when compared with that in the vehicle or OLM-treated group. These observations are consistent with our clinical study in diabetic nephropathy in which the combination of diuretics with a RAS inhibitor decreased albumin excretion and oxidative stress. ${ }^{33}$ Inhibition of renal oxidative stress by ARB may have been involved in this mechanism as indicated in this study.

In conclusion, ARB therapy is expected to have not only an antihypertensive effect but also a beneficial role in organ protection in the therapy of hypertension. This study has confirmed that ARB administration reduced the slope of the Na-MAP relationship curve and also increased salt sensitivity. Importantly, it also seems that the fear of worsening renal oxidative stress with combined HCTZ and OLM therapy may not be warranted, and that this combination may actually have a beneficial role in reducing both blood pressure and oxidative stress under conditions of a high salt intake. As a word of caution, it should also be recognized that although salt restriction is important for the antihypertensive therapy of $\mathrm{ARB}$, one should also take into account the fact that if blood pressure is markedly reduced when salt intake is suddenly restricted, acute renal failure could ensue because of dehydration and the reduction of renal blood flow, as is often seen in elderly patients. However, it seems clear that the combined treatment of ARBs with a diuretic will enhance the antihypertensive effects of ARB treatment alone and further reduce hypertension in salt-sensitive patients who fail to control their salt intake.

\section{ACKNOWLEDGEMENTS}

We thank Dr Allen W Cowley for his expert review of the paper, and Teruko Sueta for her expert assistance with animal care. This study was supported by Grants-in-Aid for Scientific Research from the Ministry of Education, Culture, Sports, Science and Technology of Japan (No. 17390245 and No. 17590181), the 21st Century COE Program, Special Research Grant from the Ministry of Education, Sports of Japan and Culture, and research grant for cardiovascular research (13C-5) from the Ministry of Health, Labour and Welfare of Japan.

1 Hansson L, Zanchetti A, Carruthers SG, Dahlöf B, Elmfeldt D, Julius S, Ménard J, Rahn $\mathrm{KH}$, Wedel $\mathrm{H}$, Westerling S. Effects of intensive blood-pressure lowering and low-dose aspirin in patients with hypertension: principal results of the Hypertension Optimal Treatment (HOT) randomised trial. Lancet 1998; 351: 1755-1762.

2 Morimoto S, Yano Y, Maki K, Sawada K. Renal and vascular protective effects of telmisartan in patients with essential hypertension. Hypertens Res 2006; 29: 567-572.

3 Dahlöf B, Devereux RB, Kjeldsen SE, Julius S, Beevers G, de Faire U, Fyhrquist F, Ibsen $\mathrm{H}$, Kristiansson K, Lederballe-Pedersen O, Lindholm LH, Nieminen MS, Omvik P, Oparil $\mathrm{S}$, Wedel H, LIFE Study Group. Cardiovascular morbidity and mortality in the Losartan Intervention For Endpoint reduction in hypertension study (LIFE): a randomised trial against atenolol. Lancet 2002; 359: 995-1003.

4 Herlitz H, Dahlof B, Jonsson O, Friberg P. Relationship between salt and blood pressure in hypertensive patients on chronic ACE-inhibition. Blood Press 1998; 7: 47-52.

5 Fang Z, Sripairojthikoon W, Calhoun DA, Zhu S, Berecek KH, Wyss JM. Interaction between lifetime captopril treatment and $\mathrm{NaCl}$-sensitive hypertension in spontaneously hypertensive rats and Wistar-Kyoto rats. J Hypertens 1999; 17: 983-991.

$6 \mathrm{Xu} \mathrm{L,} \mathrm{Brooks} \mathrm{VL.} \mathrm{Sodium} \mathrm{intake,} \mathrm{angiotensin} \mathrm{II} \mathrm{receptor} \mathrm{blockade,} \mathrm{and} \mathrm{baroreflex}$ function in conscious rats. Hypertension 1997; 29: 450-457.

7 Shimosawa T, Gohchi K, Yatomi Y, Fujita T. Effectiveness of add-on low-dose diuretics in combination therapy for hypertension: losartan/hydrochlorothiazide vs candesartan/ amlodipine. Hypertens Res 2007; 30: 831-837.

8 Weir MR, Smith DH, Neutel JM, Bedigian MP. Valsartan alone or with a diuretic or ACE inhibitor as treatment for African American hypertensives: relation to salt intake. Am J Hypertens 2001; 14: 665-671.

9 Vogt L, Waanders F, Boomsma F, de Zeeuw D, Navis G. Effects of dietary sodium and hydrochlorothiazide on the antiproteinuric efficacy of losartan. J Am Soc Nephrol 2008; 19: 999-1007.

10 Hall JE, Guyton AC, Smith Jr MJ, Coleman TG. Long-term regulation of arterial pressure, glomerular filtration and renal sodium reabsorption by angiotensin II in dogs. Clin Sci (Lond) 1980; 59: 87-90.

11 Unger T, Chung O, Csikos T, Culman J, Gallinat S, Gohlke P, Höhle S, Meffert S, Stoll M, Stroth U, Zhu YZ. Angiotensin receptors. J Hypertens Supp/ 1996; 14: S95-S103.

12 Makino A, Skelton MM, Zou AP, Cowley Jr AW. Increased renal medullary $\mathrm{H}_{2} \mathrm{O}_{2}$ leads to hypertension. Hypertension 2003; 42: 25-30.

13 Kitiyakara C, Chabrashvili T, Chen Y, Blau J, Karber A, Aslam S, Welch WJ, Wilcox CS. Salt intake, oxidative stress, and renal expression of NADPH oxidase and superoxide dismutase. J Am Soc Nephrol 2003; 14: 2775-2782.

14 Guo P, Nishiyama A, Rahman M, Nagai Y, Noma T, Namba T, Ishizawa M, Murakami K, Miyatake A, Kimura S, Mizushige K, Abe Y, Ohmori K, Kohno M. Contribution of reactive oxygen species to the pathogenesis of left ventricular failure in Dahl saltsensitive hypertensive rats: effects of angiotensin II blockade. J Hypertens 2006; 24: 1097-1104.

15 Cowley Jr AW. Long-term control of arterial blood pressure. Physiol Rev 1992; 72 : 231-300.

16 Kimura G, Saito F, Kojima S, Yoshimi H, Abe H, Kawano Y, Yoshida K, Ashida T, Kawamura M, Kuramochi M. Renal function curve in patients with secondary forms of hypertension. Hypertension 1987; 10: 11-15.

17 Cowley Jr AW, Mori T, Mattson D, Zou AP. Role of renal NO production in the regulation of medullary blood flow. Am J Physiol Regul Integr Comp Physiol 2003; 284: 1355-1369.

18 Oliverio MI, Best CF, Smithies O, Coffman TM. Regulation of sodium balance and blood pressure by the AT $(1 \mathrm{~A})$ receptor for angiotensin II. Hypertension 2000; 35: 550-554.

19 Gross V, Milia AF, Plehm R, Inagami T, Luft FC. Long-term blood pressure telemetry in AT2 receptor-disrupted mice. J Hypertens 2000; 18: 955-961.

20 Uzu T, Kimura G, Yamauchi A, Kanasaki M, Isshiki K, Araki S, Sugiomoto T, Nishio Y, Maegawa H, Koya D, Haneda M, Kashiwagi A. Enhanced sodium sensitivity and disturbed circadian rhythm of blood pressure in essential hypertension. $J$ Hypertens 2006; 24: 1627-1632.

21 Schnecko A, Witte K, Lemmer B. Effects of the angiotensin II receptor antagonist losartan on 24-h blood pressure profiles of primary and secondary hypertensive rats. J Cardiovasc Pharmacol 1995; 26: 214-221.

22 Sachdeva A, Weder AB. Nocturnal sodium excretion, blood pressure dipping, and sodium sensitivity. Hypertension 2006; 48: 527-533.

23 Hermida RC, Calvo C, Ayala DE, Fernández JR, Covelo M, Mojón A, López JE. Treatment of non-dipper hypertension with bedtime administration of valsartan. J Hypertens 2005; 23: 1913-1922. 
24 Van den Buuse M. Circadian rhythms of blood pressure, heart rate, and locomotor activity in spontaneously hypertensive rats as measured with radio-telemetry. Physiol Behav 1994; 55: 783-787.

25 Fukuda M, Yamanaka T, Mizuno M, Motokawa M, Shirasawa Y, Miyagi S, Nishio T, Yoshida A, Kimura G. Angiotensin II type 1 receptor blocker, olmesartan, restores nocturnal blood pressure decline by enhancing daytime natriuresis. J Hypertens 2008; 26: 583-588.

26 Mori T, Cowley Jr AW, Ito S. Molecular mechanisms and therapeutic strategies of chronic renal injury: physiological role of angiotensin II-induced oxidative stress in renal medulla. J Pharmacol Sci 2006; 100: 2-8.

27 Uzu T, Kimura G. Diuretics shift circadian rhythm of blood pressure from nondipper to dipper in essential hypertension. Circulation 1999; 100: 1635-1638.

28 Taylor NE, Glocka P, Liang M, Cowley Jr AW. NADPH oxidase in the renal medulla causes oxidative stress and contributes to salt-sensitive hypertension in Dahl $\mathrm{S}$ rats. Hypertension 2006; 47: 692-698.
29 Mori T, Cowley Jr AW. Angiotensin II-NAD(P)H oxidase-stimulated superoxide modifies tubulovascular nitric oxide cross-talk in renal outer medulla. Hypertension 2003; 42 : 588-593.

30 Mori T, O'Connor PM, Abe M, Cowley Jr AW. Enhanced superoxide production in renal outer medulla of Dahl salt-sensitive rats reduces nitric oxide tubular-vascular cross-talk. Hypertension 2007; 49: 1336-1341.

31 Pech V, Sikka SC, Sindhu RK, Vaziri ND, Majid DS. Oxidant stress and blood pressure responses to angiotensin II administration in rats fed varying salt diets. Am J Hypertens 2006; 19: 534-540.

32 Reungjui S, Hu H, Mu W, Roncal CA, Croker BP, Patel JM, Nakagawa T, Srinivas T, Byer K, Simoni J, Wesson D, Sitprija V, Johnson RJ. Thiazide-induced subtle renal injury not observed in states of equivalent hypokalemia. Kidney Int 2007; 72: 1483-1492.

33 Ogawa S, Mori T, Nako K, Kato T, Takeuchi K, Ito S. Angiotensin II type 1 receptor blockers reduce urinary oxidative stress markers in hypertensive diabetic nephropathy. Hypertension 2006; 47: 699-705. 